ARTICLE

Received 28 Aug 2013 | Accepted 17 Dec 2013 | Published 4 Feb 2014 DOI 10.1038/ncomms4132

\title{
A wearable and highly sensitive pressure sensor with ultrathin gold nanowires
}

\author{
Shu Gong ${ }^{1,2}$, Willem Schwalb ${ }^{3}$, Yongwei Wang ${ }^{1,2}$, Yi Chen ${ }^{1}$, Yue Tang ${ }^{1,2}$, Jye Si ${ }^{1}$, Bijan Shirinzadeh ${ }^{3}$ \\ \& Wenlong Cheng ${ }^{1,2}$
}

Ultrathin gold nanowires are mechanically flexible yet robust, which are novel building blocks with potential applications in future wearable optoelectronic devices. Here we report an efficient, low-cost fabrication strategy to construct a highly sensitive, flexible pressure sensor by sandwiching ultrathin gold nanowire-impregnated tissue paper between two thin polydimethylsiloxane sheets. The entire device fabrication process is scalable, enabling facile large-area integration and patterning for mapping spatial pressure distribution. Our gold nanowires-based pressure sensors can be operated at a battery voltage of $1.5 \mathrm{~V}$ with low energy consumption ( $<30 \mu \mathrm{W}$ ), and are able to detect pressing forces as low as $13 \mathrm{~Pa}$ with fast response time $(<17 \mathrm{~ms})$, high sensitivity $\left(>1.14 \mathrm{kPa}^{-1}\right)$ and high stability $(>50,000$ loading-unloading cycles). In addition, our sensor can resolve pressing, bending, torsional forces and acoustic vibrations. The superior sensing properties in conjunction with mechanical flexibility and robustness enabled real-time monitoring of blood pulses as well as detection of small vibration forces from music.

\footnotetext{
${ }^{1}$ Department of Chemical Engineering, Monash University, Clayton, Victoria 3800, Australia. ${ }^{2}$ The Melbourne Centre for Nanofabrication, 151 Wellington Road, Clayton, Victoria 3800, Australia. ${ }^{3}$ Mechanical and Aerospace Engineering, Faculty of Engineering, Monash University, Clayton, Victoria 3800, Australia. Correspondence and requests for materials should be addressed to W.C. (email: wenlong.cheng@monash.edu).
} 
W earable and lightweight pressure sensing devices are of paramount importance for various future applications, such as electronic skin ${ }^{1-7}$, touch-on flexible displays ${ }^{8,9}$, soft robotics ${ }^{10,11}$ and energy harvesting ${ }^{12-14}$. To date, pressure sensors are typically based on forceinduced changes in capacitance $2,8,15,16$, piezoelectricity ${ }^{17-19}$, triboelectricity 7,13 and resistivity ${ }^{20,21}$. Recently, various nanomaterials, including nanowires ${ }^{4,22,23}$, carbon nanotubes $8,15,20$, polymer nanofibers ${ }^{17,19,21,24}$, metal nanoparticles ${ }^{25-27}$ and graphene $^{28}$ have been used for the design of novel flexible pressure and strain sensors. The majority of these nanomaterialsbased pressure sensors are based on capacitance or piezoelectricity except for a few recent reports ${ }^{20,21}$, where resistivity was used for designing strain gauge sensors. The advantage of resistive pressure sensors lies at simplicity in device fabrication as well as relatively low energy consumption in operation. Despite the promise, to date, only limited successful examples were reported on resistivity-based flexible pressure sensors $^{20,21}$.

The recently developed ultrathin gold nanowires (AuNWs) $(\sim 2 \mathrm{~nm} \text { in width, with an aspect ratio of }>10,000)^{29-31}$ are mechanically flexible yet robust, enabling their uses in constructing novel superlattice nanomembranes ${ }^{32}$ and flexible transparent electrodes ${ }^{33}$. Despite superior mechanical and electrical properties, ultrathin AuNWs have not yet been used in designing flexible sensors.

Herein, we demonstrate that an AuNWs-impregnated tissue paper could be sandwiched between a blank PDMS sheet and a patterned PDMS sheet with interdigitated electrode arrays, leading to a superior wearable pressure sensor. Typically, a sensitivity of $1.14 \mathrm{kPa}^{-1}$ could be achieved, which is comparable with the record in organic transistor pressure sensors reported recently ${ }^{3}$. Furthermore, our sensor could respond to pressure changes within $0.05 \mathrm{~s}$ at a frequency up to $5.5 \mathrm{~Hz}$. We observed negligible loading-unloading signal changes over 50,000 cycles. Notably, the key sensing elements, AuNWs-impregnated tissue paper could be easily fabricated in large amount at low-cost using scalable wet chemistry processing steps. This enabled facile largearea fabrication and patterning for spatial pressure mapping. In addition, our AuNWs-based pressure sensors could be used to detect pressing, bending and torsional forces with high sensitivity, enabling their uses as wrist sensors and vibration sensors with low power consumption $(<30 \mu \mathrm{W}$ for an operating voltage of $1.5 \mathrm{~V}$ ). These performances are comparable with the recently reported pressure sensing devices ${ }^{3,5-7,13}$, simultaneously offering advantages of low-cost and simplicity in device fabrication as well as versatility of detecting various force signals.

\section{Results}

Device fabrication. Figure 1a illustrates the fabrication process of our AuNWs-based pressure sensor. First, high-quality AuNWs were synthesized following the protocol reported recently ${ }^{31}$ and concentrated to a stock solution of $\sim 11 \mathrm{mg} \mathrm{ml}^{-1}$. Note that AuNWs were ultrathin (only about $2 \mathrm{~nm}$ in width, comparable with the typical polymer chain width), yet tens of micrometres long, corresponding to an aspect ratio of $>10,000$. In addition, AuNWs were mechanically robust yet flexible, with curved structures without breaking ${ }^{32}$ (Supplementary Fig. 1).

Second, AuNWs were deposited into Kimberly-Clark tissue paper by simple dip-coating and drying. The dip-coating process a

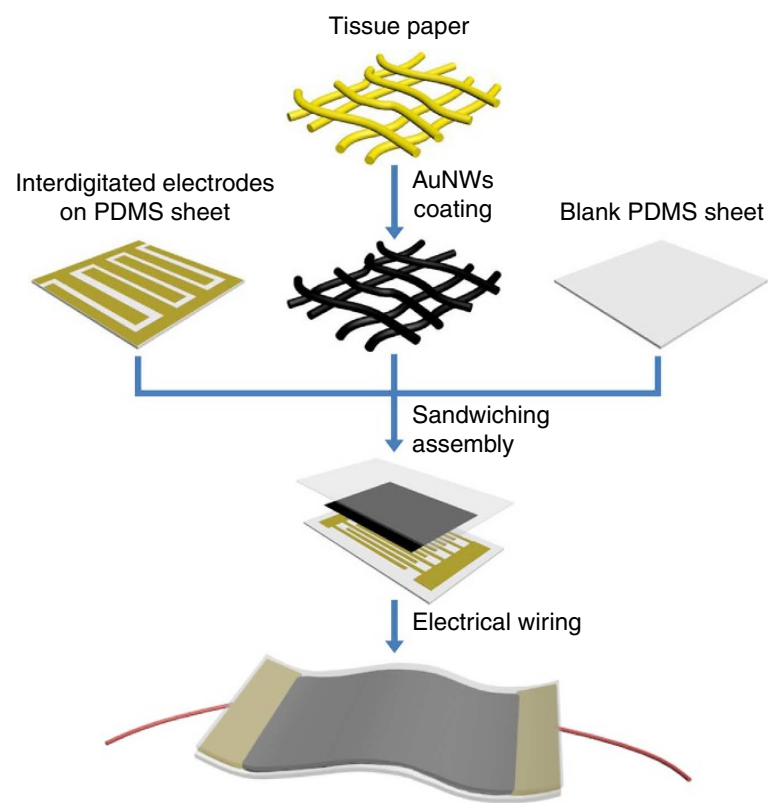

b

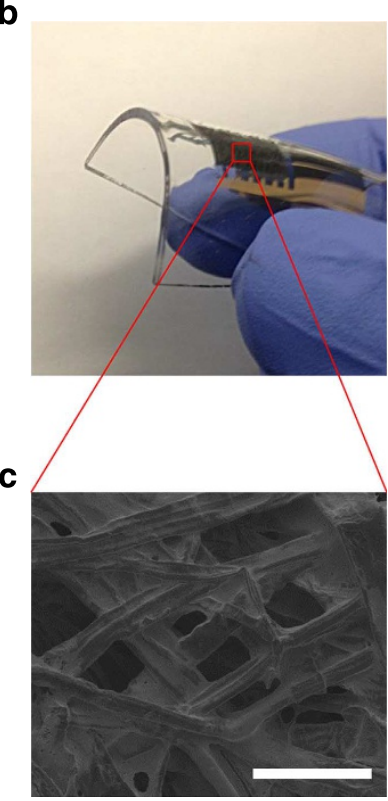

d

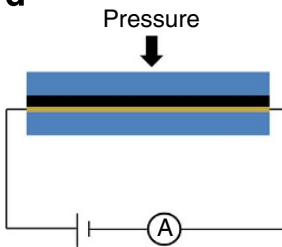

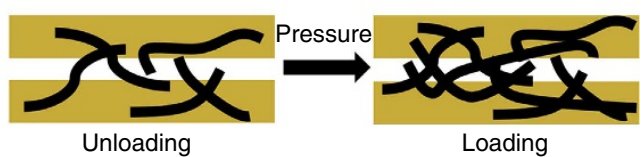

Unloading

Loading

e

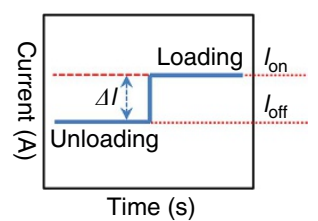

Figure 1 | Pressure sensor based on the AuNWs coated tissue paper. (a) Schematic illustration of the fabrication of a flexible sensor. (b) Photograph showing the bendability of the sensor. (c) Scanning electron microscopy image of the morphology of gold nanowires coated tissue fibres (scale bar, $100 \mu \mathrm{m}$ ). (d) Schematic illustration of the sensing mechanism. (e) Current changes in responses to loading and unloading ( $I_{\text {off: }}$ unloading, $I_{\text {on: }}$ loading). 
was repeated for 10 times, giving a dark tissue paper with a sheet resistance of $2.5 \pm 0.4 \mathrm{M} \Omega \mathrm{sq}^{-1}$ measured from 10 samples, and leading to an average off-resistance of $91.1 \pm 52 \mathrm{k} \Omega$ after their integration into pressure sensors (Supplementary Fig. 2). Both optical microscopy and scanning electron microscopy demonstrated uniform deposition of AuNWs onto tissues papers (Supplementary Fig. 3). Finally, the AuNWs-impregnated tissue paper was sandwiched between a blank $500 \mu \mathrm{m}$-thick PDMS sheet and a PDMS sheet patterned with interdigitated electrode arrays (Supplementary Fig. 4). PDMS surfaces were treated by plasma before sandwiching, enabling their conformal contact and firm bonding with tissue paper (Supplementary Fig. 5). Such fabricated devices are wearable and bendable due to the flexible nature of both tissue paper and AuNWs (Fig. 1b,c).

The sensing mechanism is due to pressing force-dependent contact between AuNWs and interdigitated electrode arrays. Unlike a bulk rigid planar metal, soft tissue paper has porous and rough surfaces with hairy AuNWs. The number of AuNWs bridging finger electrode pairs depended on the external forces applied. On applying an external pressure, a small compressive deformation of tissue paper enabled more AuNWs in contact with finger electrodes, leading to more conductive pathways (Fig. 1d). This caused an increase in current when a fixed voltage of $1.5 \mathrm{~V}$ was applied (Fig. 1e). On unloading, both PDMS and tissue paper recovered to their original shapes, reducing the amount of AuNWs bridging the finger electrode pairs, therefore, leading to the decrease of the current.

Cycling tests and sensitivity. To measure the responses of our AuNWs-based sensors to both static and dynamic mechanical pressures, a home-made system containing a computer-controlled stepping motor and a force sensor were designed (Fig. 2a). Such a system can provide an external pressure of up to $50 \mathrm{kPa}$ (static pressure) and $3 \mathrm{kPa}$ (dynamic pressure) with electrical signals simultaneously recorded. Our AuNWs-based pressure

b
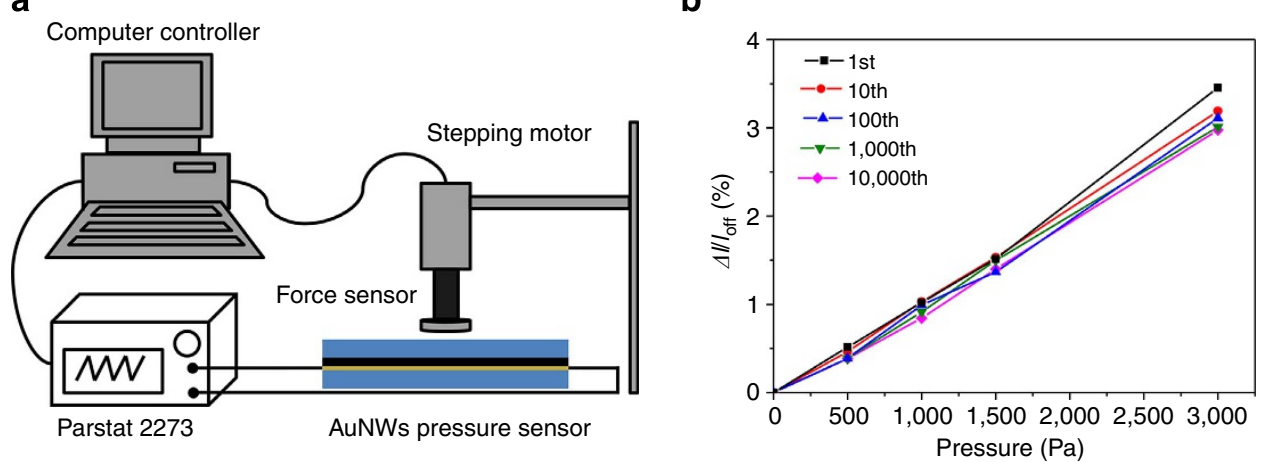

C

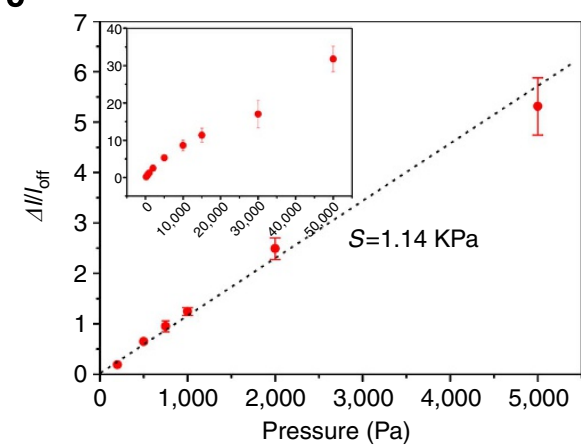

e

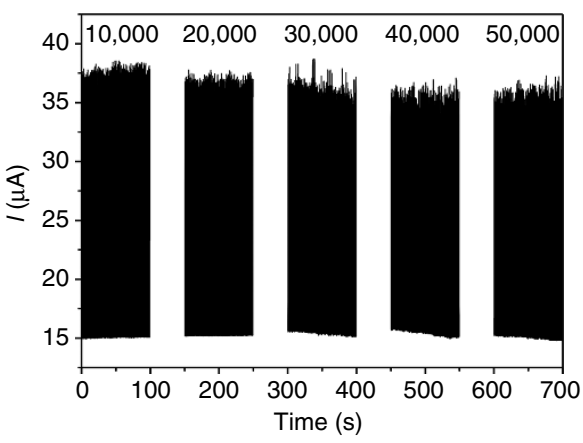

d

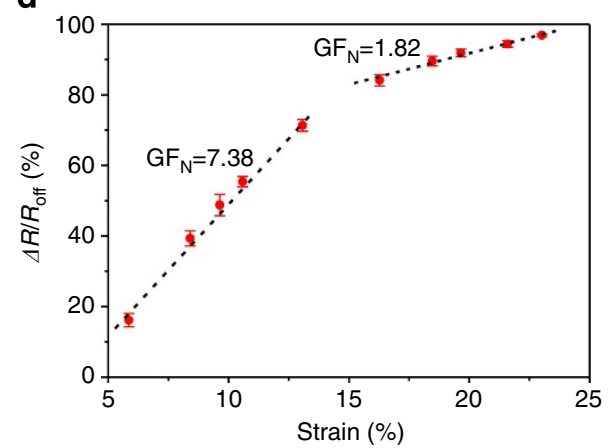

f

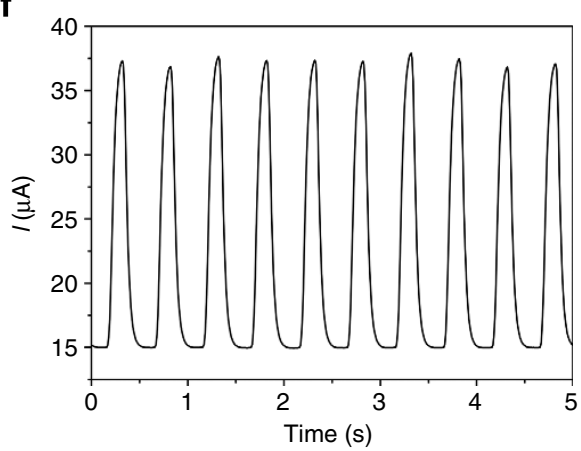

Figure 2 | Evaluation of sensing performances. (a) Schematic illustration of the experimental setup. (b) Sensitivity after repeated loading-unloading cycles at a frequency of $3 \mathrm{~Hz}$. (c) Current response to various pressures. Dot line is a linear regression giving a sensitivity of $\sim 1.14 \mathrm{kPa}$. (d) Electrical resistance changes under various strains. Gauge factor, $\mathrm{GF}_{\mathrm{N}}$ could be derived by linear fitting. (e) The durability test under a pressure of 2,500 Pa at a frequency at $2 \mathrm{~Hz}$. The current change curves were recorded after each 10,000 cycles and 200 cycles of data were presented in each recording. (f) Enlarged view of the part of the $l-t$ curve in e after 10,000 loading-unloading cycles. 
a

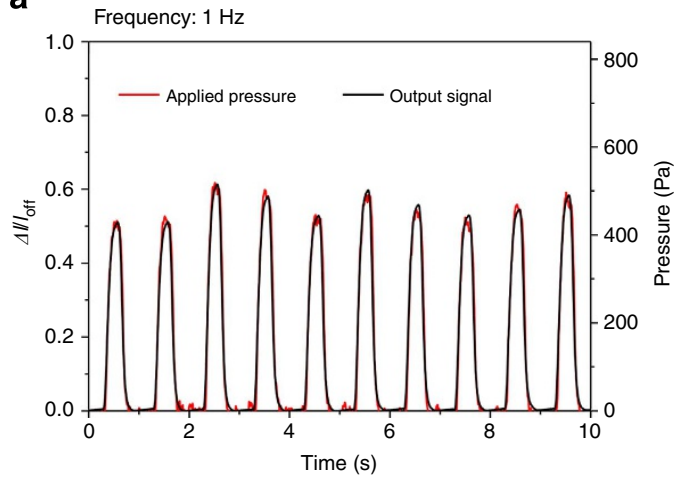

b

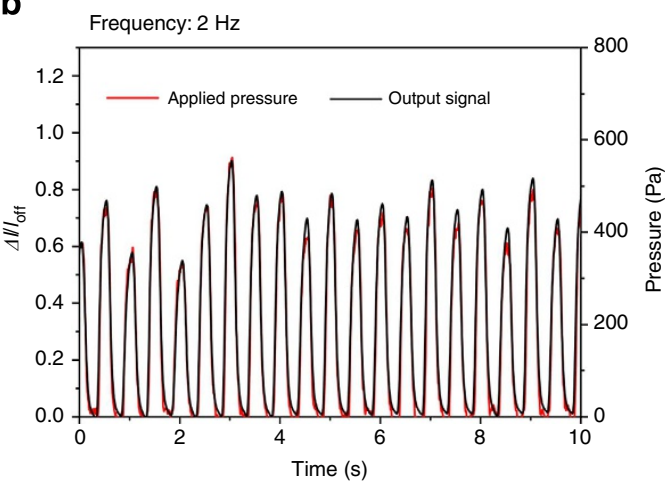

C
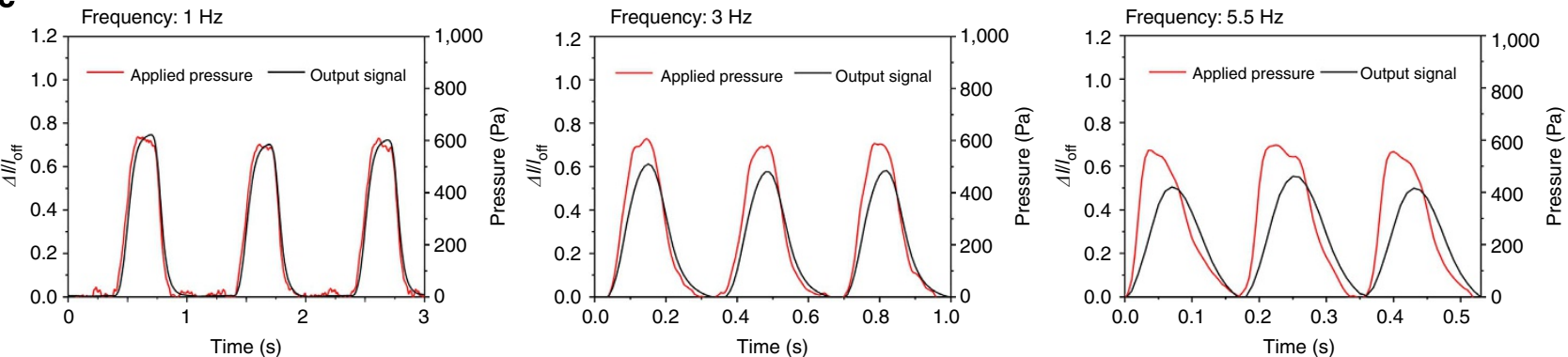

Figure 3 | Pressure-resolved and time-resolved responses. (a, b) Pressure-resolved measurements of the output signal as the function of applied pressure in the range of $400-550 \mathrm{~Pa}$ at the frequency of $1 \mathrm{~Hz}$ and $2 \mathrm{~Hz}$, respectively. (c) Plots showing frequency responses at the dynamic pressure of 600 Pa: pressure input frequency of 1,3 and $5.5 \mathrm{~Hz}$.
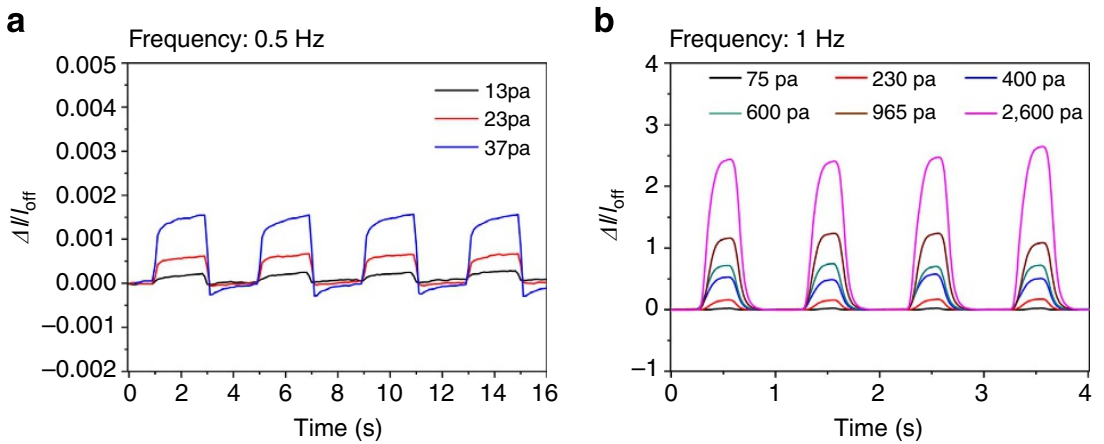

Figure 4 | Dynamic pressure response at low and high pressure range. (a) Plot of current response as a function of time (pressure input frequency: $0.5 \mathrm{~Hz}$ ) for three applied pressures $(13,23$ and $37 \mathrm{~Pa}$ ). (b) Plot of current response of the sensor as a function of time (pressure input frequency: $1 \mathrm{~Hz}$ ) for the applied pressures in the range of $75-2,600 \mathrm{~Pa}$.

sensor showed a steady response to static pressure up to $50 \mathrm{kPa}$ and the resistance under each pressure was constant (Supplementary Fig. 6). For dynamic force measurement, we performed multiple loading-unloading tests under various pressures at a frequency of $3 \mathrm{~Hz}$. After $\sim 10,000$ cycles, the sensor performance showed little changes at various pressures ranging from $500-3,000 \mathrm{~Pa}$ (Fig. 2b).

The sensitivity of our pressure sensor is defined as $S=\left(\Delta I / I_{\text {off }}\right) /$ $\Delta P$, where $\Delta I$ is the relative change in current, $I_{\text {off }}$ is current of the sensor under no load and $\Delta P$ is the change in applied pressure. An approximately linear relationship between $\Delta I / I_{\text {off }}$ and applied pressure $P$ in the range of $0-5 \mathrm{kPa}$, leading to a sensitivity value $S=1.14 \mathrm{kPa}^{-1}$. This value is only next to the record value in organic transistor pressure sensors ${ }^{3}$ and higher than the typical sensitivity of $5 \times 10^{-3}-0.55 \mathrm{kPa}^{-1}$ reported from other pressure sensors, which have a sensing area of $0.6 \times 0.6 \mathrm{~mm}^{2}-8 \times 8 \mathrm{~mm}$ (refs $22,34-37$ ). In addition, higher sensitivity could be achieved by further increasing the dipcoating cycles of AuNWs (Supplementary Fig. 7) and reducing the PDMS thickness (Supplementary Fig. 8).

To assess the ability of our device to function as a strain gauge sensor, we measured the gauge factor (GF), which is usually defined as the ratio of relative change in electrical resistance $(\Delta R /$ $\left.R_{\text {off }}\right)$ to the mechanical strain, $\epsilon$. For our case, the forces were applied at normal directions, causing the thickness changes, $\varepsilon_{\mathrm{N}}$. Then, GF at the normal direction $\mathrm{GF}_{\mathrm{N}}=\left(\Delta R / R_{\text {off }}\right) / \varepsilon_{\mathrm{N}}$. As shown in Fig. 2d, the GF below the strain of $14 \%$ showed a higher linear slope of 7.38 . In the high strain range (14-25\%), the average GF dropped to 1.82 .

We further demonstrate high durability of our AuNWs pressure sensor under a pressure of $2,500 \mathrm{~Pa}$ at a frequency of $2 \mathrm{~Hz}$ (Fig. 2e). Note that the high signal-to-noise ratios were well 
maintained and the current amplitude exhibited negligible changes after 50,000 loading-unloading cycles. The high stability was also demonstrated in a bending test and the device sensitivity did not show evident changes after repeated bending for over 5,000 cycles at $30 \mathrm{~mm}$ radius of curvature (Supplementary Fig. 9).

Time-resolved responses. To examine the response time of our sensors to external forces, the output current signals were compared with the dynamic pressure inputs at a frequency of $1-5.5 \mathrm{~Hz}$ (Fig. 3). Note that the current waves were almost the same as the input pressure waves under a pressure of $\sim 400 \mathrm{~Pa}$ at the lower frequency $(1-2 \mathrm{~Hz})$. Furthermore, the applied pressure was increased to $600 \mathrm{~Pa}$ at a frequency of 1,3 and $5.5 \mathrm{~Hz}$ (Fig. 3c). The output electrical signals remained stable without evident change in amplitude up to $5.5 \mathrm{~Hz}$. A negligible hysteresis was observed under a pressure of $600 \mathrm{~Pa}$ at $1 \mathrm{~Hz}$ (Supplementary Fig. 10a,b), which increased with the increasing frequency up to $0.05 \mathrm{~s}$ at $5.5 \mathrm{~Hz}$. This hysteresis may be attributed to elastic deformation of tissue paper during loading-unloading process and the viscoelastic effects of the PDMS layers ${ }^{21}$. However, the bandwidth and the line shapes kept almost unchanged as the force frequency increased. Similar quick response time was also observed in the abrupt unloading process from 1,600 to $0 \mathrm{~Pa}$ (Supplementary Fig. 10c), where a response time of $17 \mathrm{~ms}$ was obtained.

Pressure-resolved responses. To estimate the pressure range of our AuNWs-based sensors towards dynamic forces, a piezoelectric stepping positioner with minimum displacement of only $1 \mu \mathrm{m}$ was applied to the sensors. As shown in Fig. 4a a pressure of $13 \mathrm{~Pa}$ could be detected, which is equivalent to the weight of a water droplet $(\sim 13 \mu \mathrm{l})$ on a surface of $10 \mathrm{~mm}^{2}$. At the higher pressure range, the noise-free, stable continuous responses could be observed up to $2,600 \mathrm{~Pa}$ (Fig. $4 \mathrm{~b}$ ).

Detection of other types of mechanical forces. In addition to pressing forces, our pressure sensor can simultaneously be used to detect the bending and torsional forces. Remarkably, the response curves are characteristic for each kind of mechanical forces for the same device (Fig. 5 and Supplementary Movie 1). High signalto-noise ratios were observed in all the three types of force measurements, further demonstrating the high sensitivity of our AuNWs pressure sensor. The device also exhibited stable linear responses to various bending forces (Supplementary Fig. 11 and Supplementary Table 1).

Detection of wrist pulses. Our AuNWs-based pressure sensors are wearable and could be used to monitor the blood pressure of a human radial artery in real-time (Fig. 6a, Supplementary Fig. 12a and Supplementary Movie 2). The wrist pulses could be read out accurately under both normal condition ( $\sim 66$ beats per minute) and after physical exercise ( $\sim 88$ beats per minute) as shown in Fig. $6 \mathrm{~b}$. Note that the amplitude and frequency of pulses under two conditions were clearly different. Under normal condition, a typical radial artery pulse waveform was obtained with two clearly distinguishable peaks $\left(P_{1}\right.$ and $\left.P_{3}\right)$ and a late systolic augmentation shoulder $\left(P_{2}\right)$ (Fig. 6c). The line shape is known to be caused by constitution of the blood pressure from the left ventricle contracts and reflective wave from the lower body ${ }^{38}$. The radial augmentation index $\mathrm{AI}_{\mathrm{r}}=P_{2} / P_{1}$ is a characteristic value for arterial stiffness, which highly related to the age of people ${ }^{38}$. From a series of waveforms measured with our AuNWs-based pressure sensor, we estimated an average $\mathrm{AI}_{\mathrm{r}}$ of 0.7 , agreeing quite well with the literature data ${ }^{38}$ for a healthy 37 -year-old male. After the physical exercise, the pulse wave shapes exhibited a change in which the late systolic augmentation $\left(P_{2}\right)$ disappeared. This could
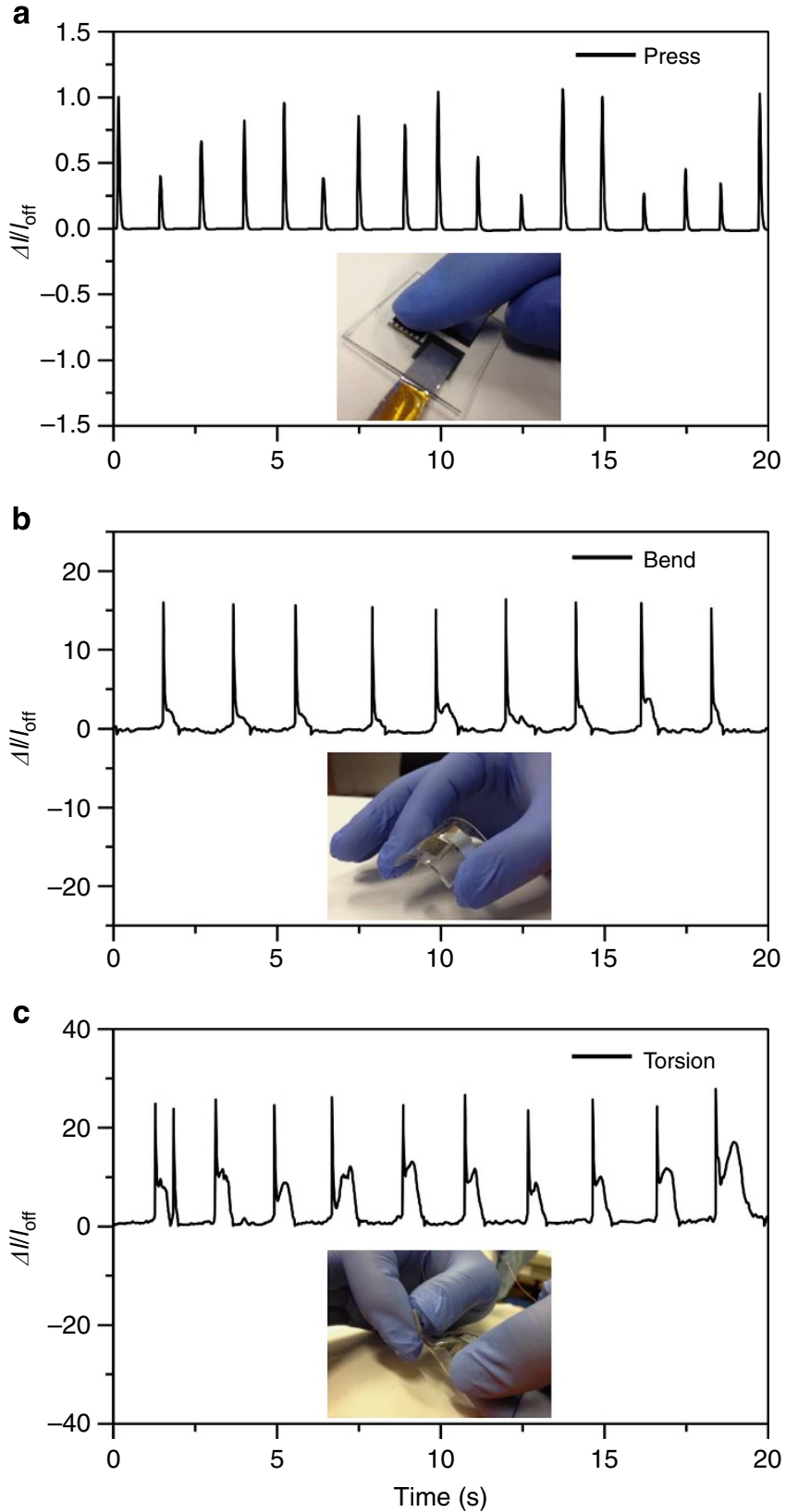

Figure 5 | Detection of other types of mechanical forces. (a-c) Plots showing the current responses to dynamic loading and unloading cycles: (a) pressing, (b) bending and (c) torsion.

be due to a few reasons such as altered heart rate/ventricular ejection characteristics, large artery stiffness/PWV, or change in tone of muscular arteries influencing pressure wave reflection as described in the literature ${ }^{39}$. The above results demonstrate that the subtle differences in blood pulses could be identified with our AuNWs-based pressure sensors, indicating its potential to serve as a wearable diagnostic device to monitor human's health in real-time under various conditions.

Detection of acoustic vibrations. Our AuNWs-based could be also applied to detect acoustic vibrations. To prove such a capability, we attached our sensor to a sponge and positioned them close to a speaker with a fixed spacing from a few to tens of millimetres (Fig. 6d). Interestingly, the tiny vibrational forces 
a

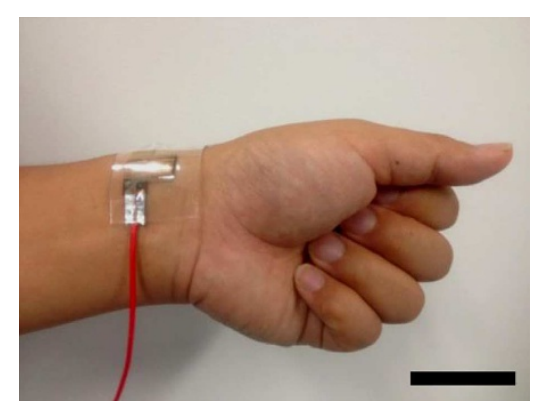

b

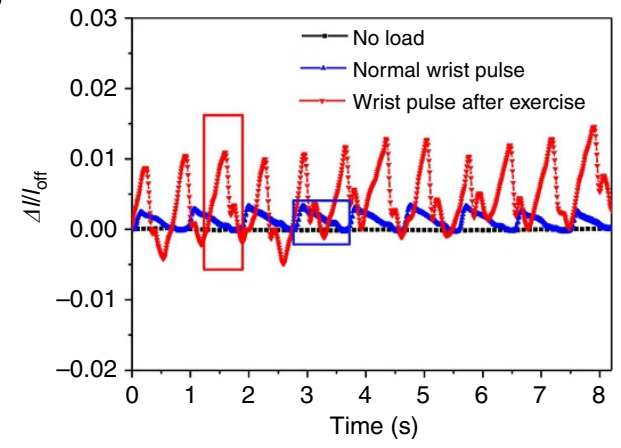

C

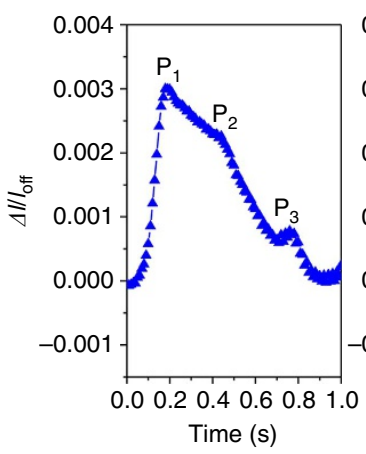

d

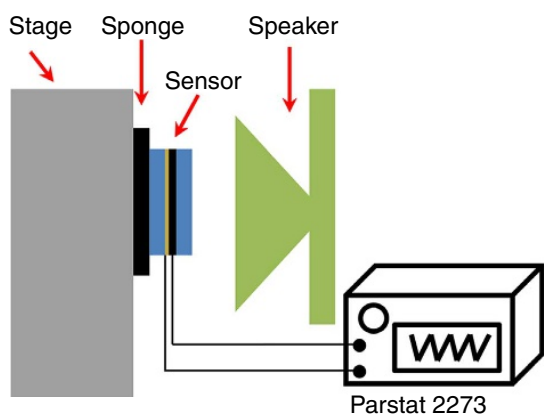

e

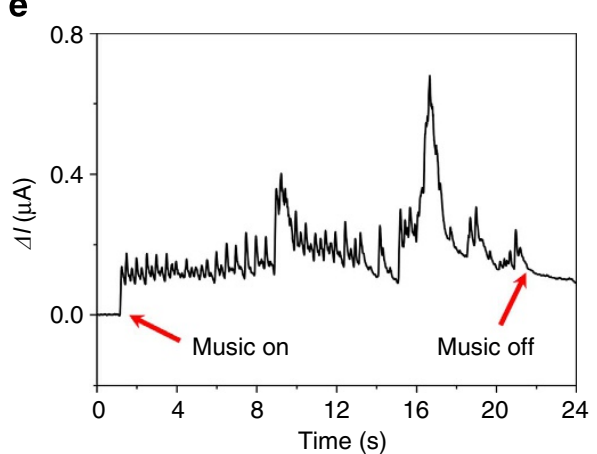

f

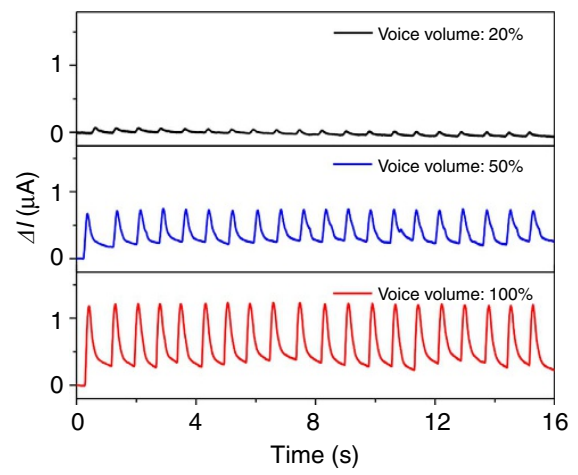

Figure 6 | Real-time and in-situ artery wrist pulses and acoustic vibrations. (a) Photograph showing the skin-attachable sensor directly above the artery of the wrist (scale bar, $3 \mathrm{~cm})$. (b, c) Measurement of the physical force of a heartbeat under normal ( 66 beats per minute) and exercise conditions (88 beats per minute). (d) Schematic illustration of the setup for acoustic vibration sensing. (e) The current responses to the acoustic vibrations from a piece of music. (f) The current responses to the acoustic vibrations from regularly clicking mouse. Note that three different voice volumes $(20,50$, and $100 \%)$ were tested.
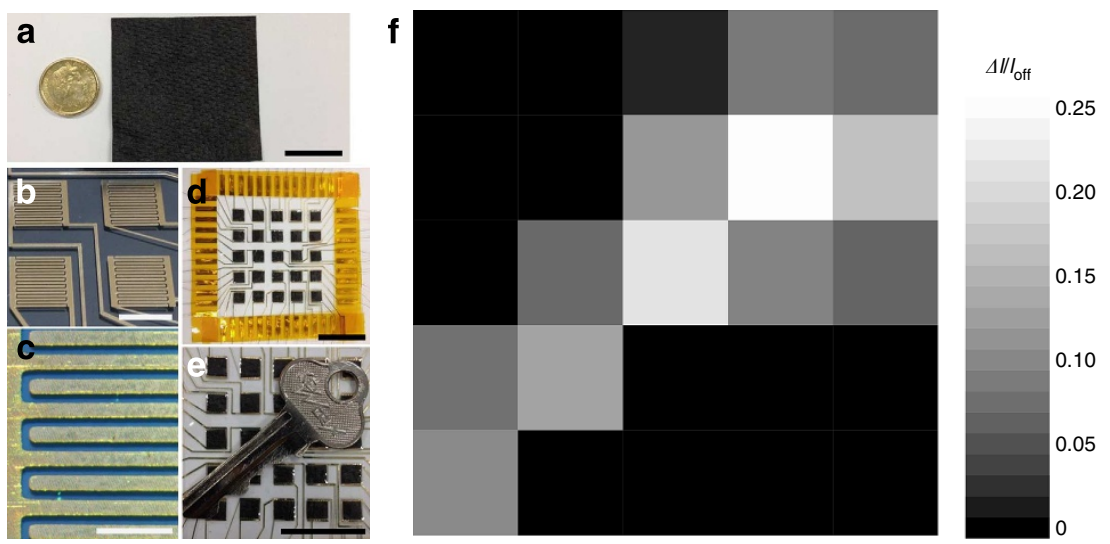

Figure 7 | Large-area integration and patterning. (a) Photograph of a $5 \times 5 \mathrm{~cm}^{2}$ gold nanowires-impregnated tissue paper (scale bar, $2 \mathrm{~cm}$ ).

(b) Photograph of interdigitated Ti-Au electrode arrays on PDMS substrate (scale bar, $5 \mathrm{~mm}$ ). (c) Optical microscope image of one pixel electrode (scale bar, $1 \mathrm{~mm}$ ). (d) Photograph of a large-area pressure sensor with $5 \times 5$ pixels (scale bar, $2 \mathrm{~cm}$ ). (e) Top view of a key $(6.6 \mathrm{~g})$ lays on the surface of the $5 \times 5$ pixel-pressure sensor (scale bar, $2 \mathrm{~cm}$ ). (f) Current mapping of pressure distributions for the key in (e). 
from both music and repeated sounds could be accurately resolved (Supplementary Fig. 12 and Supplementary Movies 3 and 4). Note that the reliable current signals accompanied to both amplitude and frequency of acoustic vibrations could be detected when the sensor was $30 \mathrm{~mm}$ away from the speaker. Even when the voice volume was decreased to $20 \%$, the well-defined current waves could still be obtained (Fig. 6f).

Large-scale integration and patterning. Both synthesis of AuNWs and fabrication of AuNWs-impregnated tissue papers are essentially based on wet chemistry techniques and both are scalable, enabling facile large-scale integration and patterning. In a typical demonstration, we fabricated an AuNWs-impregnated tissue paper sheet of $5 \times 5 \mathrm{~cm}^{2}$ (Fig. 7a) and cut them into 25 pieces $\left(4 \times 5 \mathrm{~mm}^{2}\right.$ each) and then integrated them into a $5 \times 5$ pixel arrays of sensing matrix fabricated by shadow mask lithography (Fig. $7 \mathrm{~b}, \mathrm{c})$. Each sensing pixel element was extended to two additional contact pads and connected by copper wires. When an irregular object such as a key $(6.6 \mathrm{~g})$ was positioned on the top of our sensors, the output current intensity depended on specific locations (Fig. 7e). The colour contrast mapped local pressure distribution in consistency with the shape of the key (Fig. 7f).

Generality of our fabrication strategy. We found our fabrication approach is general, which could be extended to carbon nanotubes and gold nanorods (Supplementary Fig. 13) as well. However, AuNWs showed the best sensitivity, which is perhaps due to high mechanical flexibility in conjunction with high conductivity. These attribute origins from the unique structures of AuNWs, including the ultra-thinness in width $(\sim 2 \mathrm{~nm})$ and the high aspect ratio $(>10,000)$.

\section{Discussion}

In summary, we have developed a simple yet efficient, low-cost, bottom-up approach to fabricate a wearable and highly sensitive pressure sensor using ultrathin, high-aspect-ratio AuNWs. This new sensor featured a sensitivity of $>1.14 \mathrm{kPa}^{-1}$, a fast response time of $<17 \mathrm{~ms}$, high stability over $>50,000$ cycles and a low power consumption of $<30 \mu \mathrm{W}$ when operated under a battery voltage of $1.5 \mathrm{~V}$. With this new sensor, we could detect dynamic forces in a wide pressure range $(13 \sim 50,000 \mathrm{~Pa})$ with the ability of resolving various complex forces including pressing, bending, torsion and acoustic vibrations. These attributes enabled us to monitor in real-time and in-situ the real-world force signals from radial artery blood pulses and acoustic vibrations. Notably, the entire device fabrication process is scalable without the need of complex and expensive equipment. We believe that our methodologies open a new route to low-cost pressure sensors with potential facile integration into future wearable electronics such as flexible touch-on displays, human-machine interfacing devices and prosthetic skins.

\footnotetext{
Methods

Synthesis of ultrathin AuNWs. Ultrathin gold nanowires were synthesized as described elsewhere ${ }^{24,25}$. In detail, $44 \mathrm{mg} \mathrm{HAuCl} \mathrm{H}_{4} \cdot 3 \mathrm{H}_{2} \mathrm{O}$ was added into $40 \mathrm{ml}$ hexane $(40 \mathrm{ml})$, followed by addition of $1.5 \mathrm{ml}$ Oleylamine $(\mathrm{OA})$. Note that the gold salts were not dissolved until the addition of OA, which acted as a phase-transfer reagents. After completely dissolving the gold salts, $2.1 \mathrm{ml}$ Triisopropylsilane was added into the above solution. The resulting solution was left to stand for 2 days without stirring at room temperature until the colour turned from yellow into dark red, indicating the formation of AuNWs. The residue chemicals were removed by repeated centrifugation and thorough washing by ethanol/hexane $(2 / 1, v / v)$ and finally concentrated to a $2 \mathrm{ml}$ stock solution in chloroform. For the entire process, OA molecules played a critical role in dissolving gold salts in Hexane, directing
} nanowire growth as well as stabilizing AuNWs.
Sensor fabrication. $8 \times 8 \mathrm{~mm}^{2}$ tissue papers were dipped into a chloroform solution of the AuNWs. After evaporating the chloroform, the colour of tissue paper changed from white to dark red. Then the dip-coating and drying process were repeated for about 10 cycles until the electrical resistance of paper sheets reached to $\sim 2.5 \mathrm{M} \Omega \mathrm{sq}^{-1}$. The Ti/Au interdigitated electrodes (Thickness at $3 \mathrm{~nm} / 30 \mathrm{~nm})$ were deposited onto PDMS substrates $\left(30 \times 27 \mathrm{~mm}^{2}\right)$ using a designed shadow mask by an electric beam evaporator (Intlvac Nanochrome II, $10 \mathrm{kV}$ ). The spacing between the adjacent electrodes was typically $0.1 \mathrm{~mm}$, with the width of interdigitated electrodes at $0.5 \mathrm{~mm}$. Two $10 \times 10 \mathrm{~mm}^{2}$ contact pads were deposited at the two ends of the interdigitated electrodes to establish external contacts. Then, the bottom layer electrode coated PDMS and upper layer blank PDMS supports were treated by thin oxygen plasma (Harrick Plasma Cleaner PDC-001) and permanently sealed outside the AuNWs coated tissue paper to ensure conformal contact of tissue paper and interdigitated electrodes.

Large-area fabrication and patterning. A $5 \times 5 \mathrm{~cm}^{2}$ tissue paper was first dipped into the AuNWs stock solution and dried for 10 cycles, leading to a uniform black tissue paper (Fig. 7a). Then AuNWs-impregnated paper was cut by paper knife into $4 \times 5 \mathrm{~mm}^{2}$ pieces. In the meantime, a patterned Ti/Au interdigitated electrode arrays on PDMS substrates $\left(65 \times 65 \mathrm{~mm}^{2}\right)$ was fabricated using shadow mask lithography mentioned earlier in this work (Fig. 7b,c). The spacing and width of electrodes were kept at 100 and $200 \mu \mathrm{m}$, with each interdigitated electrode pixel at $5 \times 5 \mathrm{~mm}^{2}$. Finally, each AuNWs-impregnated paper piece was addressed to the specific electrode pixels and sandwiched between the plasma-treated PDMS sheets, leading to large-area, patterned pressure sensors.

Device characterization. SEM images were characterized using a JEOL JSM$7001 F$ FEG SEM operated at $5 \mathrm{kV}$ beam voltage. TEM images were carried out using a Philips CM20 TEM at a $200 \mathrm{kV}$ accelerating voltage. Optical images were taken by a Nikon SMZ800 microscope with a Nikon Digital Sight DS-Fil camera. The sheet resistances of AuNWs coated tissue papers were carried out on a Jandel four point conductivity probe by using a linear arrayed four point head. The strains were measured by an Instron (Micro Tester, 5848, Instron) using a $100 \mathrm{~N}$ load cell and strain control mode with a strain rate of $100 \%$ per minute. The current differences and the I-V characteristics for the pressure sensor were recorded by the Parstat 2273 electrochemical system (Princeton Applied Research). For the dynamic force measurement, a computer-based user interface and a micro force sensor (ATI Nano17 Force/Torque Sensor, 1/80 N resolution without filtering) and a Maxon Brushless DC motor using a high resolution quadrature encoder $(15 \mu \mathrm{m}$ of linear resolution) were used to apply an external pressure up to 2,600 $\mathrm{Pa}$ with frequency up to $5.5 \mathrm{~Hz}$. For the dynamic low pressure measurement, a piezoelectric stepping positioner (SLC-1730) was used by a custom LabView programme and the force data was measured by an electrical balance (Mettler Toledo NewClassic MF, MS105DU).

Materials. Gold (III) chloride trihydrate $\left(\mathrm{HAuCl}_{4} \cdot 3 \mathrm{H}_{2} \mathrm{O}, \geq 99.9 \%\right)$, Triisopropylsilane (99\%) and OA were purchased from Sigma Aldrich. Ethanol, Hexane and chloroform were obtained from Merck KGaA. All chemicals were used as received unless otherwise indicated. All glassware used in the following procedures was cleaned in a bath of freshly prepared aqua regia (highly corrosive!) and rinsed thoroughly in $\mathrm{H}_{2} \mathrm{O}$ before use. Tissue papers were brought from Kimberly-Clark Worldwide Incorporation (Kleenex Brand optimum towel, $30.5 \times 24 \mathrm{~cm}^{2}$ ). PDMS substrates were made by the mixing of the prepolymer gel (Sylgard 184 Silicone Elastomer Base) and the cross linker (Sylgard 184 Silicone Elastomer Curing Agent) at the weight ratio of 10:1. The mixture was poured on a $6^{\prime \prime}$ flat-plate petri dish using $0.5 \mathrm{~mm}$-height shims as spacers and cured at $65^{\circ} \mathrm{C}$ for $2 \mathrm{~h}$ in an oven After curing, the PDMS sheet with a thickness of $500 \mu \mathrm{m}$ was cut into $30 \times 27 \mathrm{~mm}^{2}$ strips for further treatment. The stainless shadow masks were purchased from MasterCut Techniques, Australia. Silver paste was from Dupont (Dupont 4929 N, DuPont Corporation, Wilmington, DE, USA). Aqueous CNT solutions $\left(1 \mathrm{mg} \mathrm{ml}^{-1}\right)$ with sodium dodecylbenzenesulphonate (SDBS) (1:10 in quality ratio) as a surfactant were prepared according to the Hu et al. ${ }^{40}$ Gold nanorods were synthesized in accordance with our previous work ${ }^{41}$ ).

\section{References}

1. Kim, D.-H. et al. Epidermal electronics. Science 333, 838-843 (2011).

2. Mannsfeld, S. C. B. et al. Highly sensitive flexible pressure sensors with microstructured rubber dielectric layers. Nat. Mater. 9, 859-864 (2010)

3. Schwartz, G. et al. Flexible polymer transistors with high pressure sensitivity for application in electronic skin and health monitoring. Nat. Commun. 4, 1859 (2013).

4. Takei, K. et al. Nanowire active-matrix circuitry for low-voltage macroscale artificial skin. Nat. Mater. 9, 821-826 (2010).

5. Kaltenbrunner, M. et al. An ultra-lightweight design for imperceptible plastic electronics. Nature 499, 458-463 (2013).

6. Wang, C. et al. User-interactive electronic skin for instantaneous pressure visualization. Nat. Mater. 12, 899-904 (2013). 
7. Jeong, J. W. et al. Materials and optimized designs for human-machine interfaces via epidermal electronics. Adv. Mat. 25, 6776 (2013).

8. Lipomi, D. J. et al. Skin-like pressure and strain sensors based on transparent elastic films of carbon nanotubes. Nat. Nano 6, 788-792 (2011).

9. Fan, F.-R. et al. Transparent triboelectric nanogenerators and self-powered pressure sensors based on micropatterned plastic films. Nano Lett. 12, 3109-3114 (2012).

10. Someya, T. et al. A large-area, flexible pressure sensor matrix with organic field-effect transistors for artificial skin applications. Proc. Natl Acad. Sci. USA 101, 9966-9970 (2004).

11. Cheng, M., Huang, X., Ma, C. \& Yang, Y. A flexible capacitive tactile sensing array with floating electrodes. J. Micromech. Microeng. 19, 115001 (2009).

12. Wang, Z. L. \& Wu, W. Nanotechnology-enabled energy harvesting for self-powered micro-/nanosystems. Angew. Chem. Int. Ed. 51, 11700-11721 (2012).

13. Yang, Y. et al. Human skin based triboelectric nanogenerators for harvesting biomechanical energy and as self-powered active tactile sensor system. ACS Nano 7, 9213-9222 (2013).

14. $\mathrm{Hu}, \mathrm{Y}$. et al. Triboelectric nanogenerator built on suspended $3 \mathrm{~d}$ spiral structure as vibration and positioning sensor and wave energy harvester. ACS Nano 7, 10424-10432 (2013).

15. Cohen, D. J., Mitra, D., Peterson, K. \& Maharbiz, M. M. A. Highly elastic, capacitive strain gauge based on percolating nanotube networks. Nano Lett. 12, 1821-1825 (2012).

16. Matsuzaki, R., Keating, T., Todoroki, A. \& Hiraoka, N. Rubber-based strain sensor fabricated using photolithography for intelligent tires. Sensor Actuat. A Phys. 148, 1-9 (2008)

17. Gao, Q., Meguro, H., Okamoto, S. \& Kimura, M. Flexible tactile sensor using the reversible deformation of poly (3-hexylthiophene) nanofiber assemblies. Langmuir 28, 17593-17596 (2012).

18. Zhou, J. et al. Flexible piezotronic strain sensor. Nano Lett. 8, 3035-3040 (2008).

19. Mandal, D., Yoon, S. \& Kim, K. J. Origin of piezoelectricity in an electrospun poly (vinylidene fluoride-trifluoroethylene) nanofiber web-based nanogenerator and nano-pressure sensor. Macromol. Rapid Commun. 32, 831-837 (2011).

20. Yamada, T. et al. A stretchable carbon nanotube strain sensor for humanmotion detection. Nat. Nano 6, 296-301 (2011).

21. Pang, C. et al. A flexible and highly sensitive strain-gauge sensor using reversible interlocking of nanofibres. Nat. Mater. 11, 795-801 (2012).

22. Xiao, X. et al. High-Strain sensors based on $\mathrm{ZnO}$ nanowire/polystyrene hybridized flexible films. Adv. Mater. 23, 5440-5444 (2011).

23. Lin, L. et al. Triboelectric active sensor array for self-powered static and dynamic pressure detection and tactile imaging. ACS Nano 7, 8266-8274 (2013).

24. Park, M., Im, J., Park, J. \& Jeong, U. Micropatterned stretchable circuit and strain sensor fabricated by lithography on an electrospun nanofiber mat. ACS Appl. Mater. Interfaces 5, 8766-8771 (2013).

25. Maheshwari, V. \& Saraf, R. F. High-resolution thin-film device to sense texture by touch. Science 312, 1501-1504 (2006).

26. Segev-Bar, M., Landman, A., Nir-Shapira, M., Shuster, G. \& Haick, H. Tunable touch sensor and combined sensing platform: toward nanoparticle-based electronic skin. ACS Appl. Mater. Interfaces 5, 5531-5541 (2013).

27. Sangeetha, N. M., Decorde, N., Viallet, B., Viau, G. \& Ressier, L. Nanoparticlebased strain gauges fabricated by convective self assembly: strain sensitivity and hysteresis with respect to nanoparticle sizes. J. Phys. Chem. C 117, 1935-1940 (2013).

28. Yao, H. B. et al. A flexible and highly pressure-sensitive graphene-polyurethane sponge based on fractured microstructure design. Adv. Mater. 25, 6692-6698 (2013).
29. Lu, X., Yavuz, M. S., Tuan, H.-Y., Korgel, B. A. \& Xia, Y. Ultrathin gold nanowires can be obtained by reducing polymeric strands of oleylamine- $\mathrm{AuCl}$ complexes formed via aurophilic interaction. J. Am. Chem. Soc. 130, 8900-8901 (2008).

30. Huo, Z., Tsung, C.-k., Huang, W., Zhang, X. \& Yang, P. Sub-two nanometer single crystal Au nanowires. Nano Lett. 8, 2041-2044 (2008).

31. Feng, H. et al. Simple and rapid synthesis of ultrathin gold nanowires, their selfassembly and application in surface-enhanced Raman scattering. Chem. Commun. 1984-1986 (2009).

32. Chen, Y., Ouyang, Z., Gu, M. \& Cheng, W. Mechanically strong, optically transparent, giant metal superlattice nanomembranes from ultrathin gold nanowires. Adv. Mater. 25, 80-85 (2013).

33. Sánchez-Iglesias, A. et al. Highly transparent and conductive films of densely aligned ultrathin au nanowires monolayers. Nano Lett. 12, 6066-6070 (2012)

34. Liu, X. et al. A highly sensitive pressure sensor using a Au-patterned polydimethylsiloxane membrane for biosensing applications. J Micromech. Microeng. 23, 025022 (2013).

35. Lee, D.-W. \& Choi, Y.-S. A novel pressure sensor with a PDMS diaphragm. Microelectron. Eng. 85, 1054-1058 (2008).

36. Chen, P.-J., Rodger, D. C., Saati, S., Humayun, M. S. \& Tai, Y.-C. Microfabricated implantable parylene-based wireless passive intraocular pressure sensors. J. Microelectromech. Syst. 17, 1342-1351 (2008).

37. Lee, H.-K., Chang, S.-I. \& Yoon, E. A flexible polymer tactile sensor: fabrication and modular expandability for large area deployment. J. Microelectromech. Syst 15, 1681-1686 (2006).

38. Nichols, W. W. Clinical measurement of arterial stiffness obtained from noninvasive pressure waveforms. Am. J. Hypertens 18, 3S-10S (2005).

39. Munir, S. et al. Exercise reduces arterial pressure augmentation through vasodilation of muscular arteries in humans. Am. J. Physiol. Heart Circ. Physiol. 294, H1645-H1650 (2008).

40. Hu, L. et al. Highly conductive paper for energy-storage devices. Proc. Nat Acad. Sci. 106, 21490-21494 (2009).

41. Tang, Y., Ng, K. C., Chen, Y. \& Cheng, W. Lightweight, flexible, nanorod electrode with high electrocatalytic activity. Electrochem. commun. 27, 120-123 (2012).

\section{Acknowledgements}

This work is financially supported by ARC discovery project DP120100170. This work was performed in part at the Melbourne Centre for Nanofabrication (MCN) in the Victorian Node of the Australian National Fabrication Facility (ANFF). S.G. would like to thank the financial aid from Chinese Scholarship Council (CSC)

\section{Author contributions}

W.L.C. and S.G. conceived and designed the experiments. S.G., W.S., Y.W., Y.C., Y.T. and J.S. carried out the experiments. S.G., W.L.C., Y.W., Y.C., Y.T., J.S. and B.S. analysed the data and/or discussed the results. S.G. and W.C. cowrote the paper. All authors commented on the manuscript.

\section{Additional information}

Supplementary Information accompanies this paper at http://www.nature.com/ naturecommunications

Competing financial interests: The authors declare no competing financial interests.

Reprints and permission information is available online at http://npg.nature.com/ reprintsandpermissions/

How to cite this article: Gong, S. et al. A wearable and highly sensitive pressure sensor with ultrathin gold nanowires. Nat. Commun. 5:3132 doi: 10.1038/ncomms4132 (2014). 